\title{
TORSION OF A CIRCULAR TUBE WITH LONGITUDINAL CIRCULAR HOLES*
}

\author{
BY \\ CHIH-BING LING \\ California Institute of Technology
}

Introduction. This paper presents a solution of St. Venant's torsion problem of a circular tube having a ring of uniformly distributed longitudinal circular holes of equal radii. The investigation of the stress distribution in such a tube is not only of theoretical interest but also of practical importance. The solution is found by constructing three series of harmonic functions which are invariant with respect to rotation about the axis of tube: The parametric coefficients attached to these functions are determined so as to satisfy the required boundary conditions. The case in which the central hole does not exist is also investigated. Finally, the solution is illustrated by working out several numerical examples.

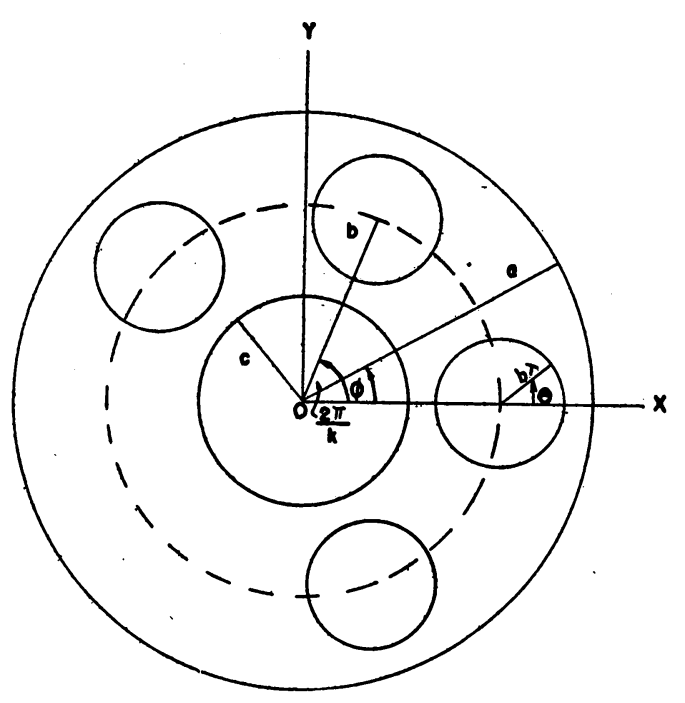

Fig. 1. Cross section of tube.

It may be mentioned that the torsion problem corresponding to the case without a central hole was solved by $M$. Kondo a few years ago by using the so-called stress distribution method. ${ }^{1}$ The same method has been used by Th. v. Kármán with success to find the potential flow around an airship, but its application to the torsion problem by Kondo seems to result in great complication. In Kondo's paper, no formula for the torsional stiffness is given.

Method of solution. Consider a circular tube defined by the exterior and the interior radii $a$ and $c$ respectively. Let there be a ring of longitudinal circular holes parallel to axis of the tube, each of radius $b \lambda$ with their centers uniformly distributed over the circumference of a concentric circle of radius $b$ at points represented in the Argand plane by

$$
z=b e^{2 m \pi i / k} \text {, }
$$

where $b$ is the distance from the center of the tube to the center of any eccentric hole, $k$ is the number of eccentric holes and $m=0,1,2, \cdots(k-1)$; the origin being at the center of the tube..Fig. 1 shows the cross section of the tube.

Define the following two systems of polar coordinates

$$
z=x+i y=a r e^{i \phi}, \quad b \zeta=z-b=b \rho e^{i \theta},
$$

where $a r, \varphi$ are polar coordinates referred to the center of the tube as origin and $b \rho, \theta$

* Received Sept. 18, 1946.

1 Masaiti Kondo, The stresses in a twisted circular cylinder having circular holes. Phil. Mag. (7) 22, 1089-1108 (1936). 
are polar coordinates referred to the center of one of the eccentric holes as origin, $r, \rho$ being dimensionless. Also, for brevity; denote the ratios of the radii by

$$
p=\frac{b}{a}, \quad q=\frac{c}{a}, \quad \cdot(q<p<1)
$$

where $a$ is the radius of the exterior boundary of the tube and $c$ is the radius of the central hole.

It is observed that the solution of St. Venant's torsion problem of the tube in question requires a harmonic function $\psi$, with its conjugate function single-valued, which satisfies the following three boundary conditions:

(1) on the exterior boundary of the tube where $|z|=a$

$$
\Psi=\psi-\left(x^{2}+y^{2}\right) / 2=0 ;
$$

(2) on the boundary of the central hole where $|z|=c$

$$
\Psi=\text { const. }=a^{2} \Psi_{1}
$$

(3) on the boundaries of the ring of eccentric holes $\rho=\lambda$

$$
\Psi=\text { const. }=a^{2} \Psi_{2},
$$

where $b \lambda$ is the radius of the eccentric hole under consideration, $\lambda$ being a dimensionless quantity. Besides, by symmetry, the function $\psi$ must be invariant with respect to rotation about the origin through angles $2 m \pi / k$ and must be even with respect to the lines of symmetry.

Hence we write

$$
\psi=\psi_{1}+\psi_{2}+\psi_{3}
$$

and construct

$$
\begin{aligned}
& \psi_{1}=a^{2} \sum_{n=0}^{\infty} A_{u} r^{n k} \cos n k \phi, \\
& \psi_{2}=a^{2} \sum_{n=1}^{\infty} \frac{B_{n}}{r^{n k}} \cos n k \phi, \\
& \psi_{3}=a^{2} \sum_{s=1}^{\infty} C_{s} U_{s},
\end{aligned}
$$

where the quantities $A, B$ and $C$ are dimensionless parametric coefficients to be adjusted so as to satisfy the required boundary conditions. The class of harmonic functions $U_{s}$, first obtained by R. C. J. Howland ${ }^{2}$ is defined by

$$
\begin{aligned}
& W_{0}=U_{0}-i V_{0}=-\log \left(z^{k}-b^{k}\right), \\
& W_{s}=U_{s}-i V_{s}=\frac{b^{s}}{(s-1) !} \frac{d^{s} W_{0}}{d b^{s}} .
\end{aligned}
$$

A brief account of these functions will be found in Appendix A. Note that in the last

2 R. C. J. Howland, Potential functions with periodicity in one coordinate, Proc. Camb. Phil. Soc. 30, 315-326 (1934). 
Eq. (8) the function $U_{0}$ is rejected on the ground that it gives rise to a multi-valued conjugate function.

Now, to apply the first and the second boundary conditions, we first express 4 in terms of the polar coordinates $(r, \phi)$ referring to the center of the tube as origin. The expansion of $W_{0}$ into a power series is different according as $|z|$ is greater or smaller than $b$. When $|z|>b$,

$$
W_{0}=-k \log z+\sum_{n=1}^{\infty} \frac{1}{n}\left(\frac{b}{z}\right)^{n k},
$$

and when $|z|<b$,

$$
W_{0}=\pi i-k \log b+\sum_{n=1}^{\infty} \frac{1}{n}\left(\frac{z}{b}\right)^{n k} .
$$

Differentiation gives

$$
\begin{aligned}
& W_{s}=k \sum_{n=1}^{\infty}\left(\begin{array}{c}
n k-1 \\
s-1
\end{array}\right)\left(\frac{b}{z}\right)^{n k}, \\
& W_{s}=(-1)^{s} k \sum_{n=0}^{\infty}\left(\begin{array}{c}
n k+s-1 \\
s-1
\end{array}\right)\left(\frac{z}{b}\right)^{n k},
\end{aligned}
$$

respectively. It is noted that the binomial coefficient $\left(\begin{array}{l}n \\ s\end{array}\right)$ vanishes when $s>n$. Taking the real part and observing that $p=b / a$, we have

$$
\begin{aligned}
& U_{s}=k \sum_{n=1}^{\infty}\left(\begin{array}{c}
n k-1 \\
s-1
\end{array}\right)\left(\frac{p}{r}\right)^{n k} \cos n k \phi, \\
& U_{s}=(-1)^{s} k \sum_{n=0}^{\infty}\left(\begin{array}{c}
n k+s-1 \\
s-1
\end{array}\right)\left(\frac{r}{p}\right)^{n k} \cos n k \phi .
\end{aligned}
$$

Hence the last Eq. (8) becomes

$$
\left.\begin{array}{l}
\psi_{3}=a^{2} \sum_{n=1}^{\infty} \frac{P_{n}}{r^{n k}} \cos n k \phi, \text { when }|z|>b \\
\psi_{3}=a^{2} \sum_{n=0}^{\infty} Q_{n} r^{n k} \cos n k \phi, \text { when }|z|<b
\end{array}\right\}
$$

where

$$
\begin{aligned}
& P_{n}=k p^{n k} \sum_{s=1}^{n k}\left(\begin{array}{c}
n k-1 \\
s-1
\end{array}\right) C_{s}, \\
& Q_{n}=\frac{k}{p^{n k}} \sum_{s=1}^{\infty}(-1)^{\circ}\left(\begin{array}{c}
n k+s-1 \\
s-1
\end{array}\right) C_{s} .
\end{aligned}
$$

Applying the boundary condition (4) at $|z|=a$ or $r=1$ and using the first series for $\psi_{3}$, we find

$$
0=A_{0}-\frac{1}{2}+\sum_{n=1}^{\infty}\left(A_{n}+B_{n}+P_{n}\right) \cos n k \phi .
$$


This relation is satisfied, provided that the coefficient of each term vanishes identically, i.e., that

$$
A_{0}-\frac{1}{2}=0, \quad A_{n}+B_{n}+P_{n}=0 .
$$

Applying the boundary condition (5) at $|z|=c$ or $r=q$ and using the second series for $\psi_{3}$, we obtain

$$
\Psi_{1}=A_{0}+Q_{0}-q^{2} / 2+\sum_{n=1}^{\infty}\left(A_{n} q^{n k}+B_{n} / q^{n k}+Q_{n} q^{n k}\right) \cos n k \phi .
$$

Equating the constants from both sides and setting each coefficient equal to zero, we find

$$
A_{0}+Q_{0}-q^{2} / 2=\Psi_{1}, \quad A_{n} q^{n k}+B_{n} / q^{n k}+Q_{n} q^{n k}=0 .
$$

To apply the third boundary condition, we first transform $\Psi$ in terms of the polar coordinates $(\rho, \theta)$ referring to the center at $(x, y)=(b, 0)$ as origin. Thus,

$$
x^{2}+y^{2}=b^{2}\left(1+\rho^{2}+2 \rho \cos \theta\right)
$$

and

$$
\begin{aligned}
r^{n k} \cos n k \phi & =R(z / a)^{n k}=p^{n k} R\left(1+\rho e^{i \theta}\right)^{n k}=p^{n k} \sum_{m=0}^{n k}\left(\begin{array}{c}
n k \\
m
\end{array}\right) \rho^{m} \cos m \theta, \\
\frac{\cos n k \phi}{r^{n k}} & =R\left(\frac{a}{z}\right)^{n k}=\frac{1}{p^{n k}} R\left(1+\rho e^{i \theta}\right)^{-n k} \\
& =\frac{1}{p^{n k}} \sum_{m=0}^{\infty}(-1)^{m}\left(\begin{array}{c}
n k+m-1 \\
m
\end{array}\right) \rho^{m} \cos m \theta .
\end{aligned}
$$

Consequently, the first two Eq. (8) become

$$
\psi_{1}=a^{2} \sum_{m=0}^{\infty} M_{m} \rho^{m} \cos m \theta, \quad \psi_{2}=a^{2} \sum_{m=0}^{\infty} N_{m} \rho^{m} \cos m \theta,
$$

where

$$
M_{m}=\sum_{n=0}^{\infty}\left(\begin{array}{c}
n k \\
m
\end{array}\right) p^{n k} A_{n}, \quad N_{m}=(-1)^{m} \sum_{n=1}^{\infty}\left(\begin{array}{c}
n k+m-1 \\
m
\end{array}\right) B_{n} / p^{n k}
$$

Again, according to Appendix A, Eq. (A.12),

$$
U_{s}=\cos s \theta / \rho^{s}+\sum_{n=0}^{\infty}(-1)^{n}{ }^{n} \alpha_{s} \rho^{n} \cos n \theta .
$$

Thus,

$$
\psi_{3}=a^{2}\left\{L_{0}+\sum_{m=1}^{\infty}\left(C_{m} / \dot{\rho}^{m}+L_{m} \rho^{m}\right) \cos m \theta\right\}
$$

where

$$
L_{m}=(-1)^{m} \sum_{s=1}^{\infty}{ }^{m} \alpha_{s} C_{s}
$$


Applying the boundary condition (6) at $\rho=\lambda$, we obtain

$$
\begin{aligned}
\Psi_{2}= & M_{0}+N_{0}+L_{0}-p^{2}\left(1+\lambda^{2}\right) / 2-p^{2} \lambda \cos \theta \\
& +\sum_{m=1}^{\infty}\left(M_{m} \lambda^{m}+N_{m} \lambda^{m}+C_{m} / \lambda^{m}+L_{m} \lambda^{m}\right) \cos m \dot{\theta} .
\end{aligned}
$$

Equating the constants and setting each coefficient equal to zero, we find

$$
\begin{aligned}
M_{0}+N_{0}+L_{0}-p^{2}\left(1+\lambda^{2}\right) / 2 & =\Psi_{2}, \\
M_{1} \lambda+N_{1} \lambda+C_{1} / \lambda+L_{1} \lambda-p^{2} \lambda & =0, \\
M_{m} \lambda^{m}+N_{m} \lambda^{m}+C_{m} / \lambda^{m}+L_{m} \lambda^{m} & =0 .
\end{aligned}
$$

From the first Eqs. (15), (16) and (22), we see that

$A_{0}=1 / 2, \quad \Psi_{1}=Q_{0}+\left(1-q^{2}\right) / 2, \quad \Psi_{2}=M_{0}+N_{0}+L_{0}-p^{2}\left(1+\lambda^{2}\right) / 2$

$\Psi_{1}$ and $\Psi_{2}$ are thus determined, if the parametric coefficients can be found. The system of simultaneous linear equations represented by the other equations is just sufficient for the determination of all parametric coefficients involved. They may be rewritten as follows:

$$
\begin{aligned}
& A_{n}+B_{n}+P_{n}=0, \quad A_{n}+q^{-2 n k} B_{n}+Q_{n}=0, \\
& \lambda^{-2} C_{1}+M_{1}+N_{1}+L_{1}=p^{2}, \quad \lambda^{-2 m} C_{m}+M_{m}+N_{m}+L_{m}=0 .
\end{aligned}
$$

The first two equations give, for $n \geqq 1$,

$$
A_{n}=\left(q^{2 n k} Q_{n}-P_{n}\right) /\left(1-q^{2 n k}\right), \quad B_{n}=q^{2 n k}\left(P_{n}-Q_{n}\right) /\left(1-q^{2 n k}\right) .
$$

Substituting these values into $M_{n}$ and $N_{n}$ in the last two equations, we find with the aid of (19), (14) and (21)

$$
C_{1}=p^{2} \lambda^{2}+\lambda^{2} \sum_{s=1}^{\infty} \beta_{s} C_{s}, \quad C_{m}=\lambda^{2 m} \sum_{s=1}^{\infty}{ }^{m} \beta_{s} C_{s},
$$

where, in general,

$$
\begin{aligned}
& { }^{m} \beta_{s}=-(-1)^{m m} \alpha_{s} \\
& +k \sum_{n=1}^{\infty}\left\{\frac{p^{2 n k}}{1-q^{2 n k}}\left(\begin{array}{c}
n k \\
m
\end{array}\right)\left[\left(\begin{array}{c}
n k-1 \\
s-1
\end{array}\right)-(-1)^{s}\left(\frac{q}{p}\right)^{2 n k}\left(\begin{array}{c}
n k+s-1 \\
s-1
\end{array}\right)\right]\right. \\
& \left.+\frac{(-1)^{m} q^{2 n k}}{\left(1-q^{2 n k}\right) p^{2 n k}}\left(\begin{array}{c}
n k+m-1 \\
m
\end{array}\right)\left[(-1)^{s}\left(\begin{array}{c}
n k+s-1 \\
s-1
\end{array}\right)-p^{2 n k}\left(\begin{array}{c}
n k-1 \\
s-1
\end{array}\right)\right]\right\} \text {. }
\end{aligned}
$$

This system of linear equations can be solved by successive approximations as follows. Write

$$
C_{m}=\sum_{t=0}^{\infty} C_{m}^{(t)}
$$

where

$$
C_{1}^{(0)}=p^{2} \lambda^{2}, \quad C_{m}^{(0)}=0,
$$


and, by iteration,

$$
C_{m}^{(t)}=\lambda^{2 m} \sum_{s=1}^{\infty}{ }^{m} \beta_{s} C_{s}^{(t-1)}
$$

With the values of $C_{m}$ thus found, the coefficients $A_{n}$ and $B_{n}$ can be obtained without difficulty from (25) with the aid of (14).

It is to be observed that the validity of the solution by successive approximations depends upon the convergence of the series (28). From physical considerations alone, it seems likely that there will be convergence as long as the boundaries do not overlap, i.e.,

$$
q \cdot+p \lambda<p<1-p \lambda, \quad \lambda<\sin (\pi / k) \text {. }
$$

To establish convergence of such a series analytically is usually difficult. However, we may proceed by first using some inequalities for the coefficients so that the series (29) can be summed, and then applying the ratio test to ascertain the range of convergence. Obviously some convergence is lost in thus using the inequalities, but it is of ten possible to establish a considerable range of convergence in this way. In the interest of brevity, no details will be given here.

Torsional stiffness. The torsional stiffness of the tube is given by

$$
H=2 \iint \Psi d x d y+2 \pi c^{2} a^{2} \Psi_{1}+2 k \pi \lambda^{2} b^{2} a^{2} \Psi_{2}
$$

where the double integration is extended over the entire cross-sectional area of the tube, excluding the holes. Let

$$
H_{j}=2 \iint \psi_{j} d x d y, \quad(j=1,2,3), \quad H_{4}=\iint\left(x^{2}+y^{2}\right) d x d y .
$$

Then,

$$
2 \iint \Psi d x d y=H_{1}+H_{2}+H_{3}-H_{4} .
$$

These integrals, save $H_{3}$, can be evaluated by ordinary methods of integration without difficulty. The following results are obtained:

$$
\begin{aligned}
& H_{1}=\pi a^{4}\left(1-q^{2}-k p^{2} \lambda^{2}\right), \quad H_{2}=0, \\
& H_{4}=\pi a^{4}\left\{1-q^{4}-k p^{4} \lambda^{2}\left(2+\lambda^{2}\right)\right\} / 2 .
\end{aligned}
$$

The integration of $\mathrm{H}_{3}$ by ordinary methods fails since there exist singularities at the centers of the eccentric holes. However, $H_{3}$ can be evaluated by using the following relation derived from Green's theorem, in which $F$ is a function of the complex variable $z$ regular in the domain $S$ enclosed by a contour $C$.

$$
\iint_{S} \frac{d F}{d z} d S=\frac{1}{2} i \int_{C} F d \bar{z},
$$

the contour $C$ being taken in a counter-clockwise direction. In this equation set $W_{s}=d F / d z$, so that 


$$
F=\int^{z} W_{s} d z
$$

and let the contour $C$ be taken around the sector of $1 / 2 k$ of the cross section, as shown in Fig. 2. Then

$$
\iint U_{s} d x d y=2 k R \iint_{S} W_{s} d S=k R i \int_{C} F d \bar{z} .
$$

Using the appropriate series of $W_{8}$ in different parts of the contour, we find that this integral vanishes identically for all values of $s$ except for $s=1$. The term arising from $1 / \zeta$ in $W_{1}$ does not vanish when the integration is taken along the semicircle $\rho=\lambda$. Here

$$
F=\int \frac{d z}{\zeta}=b \log \zeta,
$$

and when $\rho=\lambda$,

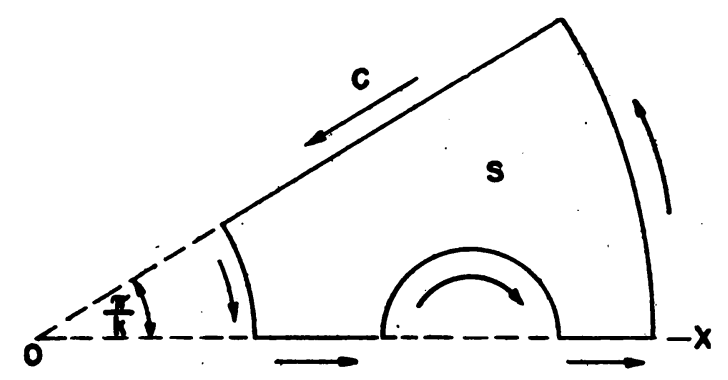

Fig. 2. Contour $C$ of $1 / 2 k$ of section.

$$
F=b(\log \lambda+i \theta), \quad d \bar{z}=b \lambda d e^{-i \theta} .
$$

We thus find

$$
\int_{\pi}^{0} F d \bar{z}=b^{2} \lambda(2+2 \log \lambda+i \pi) .
$$

Hence,

$$
\iint U_{1} d x d y=-\pi k \lambda b^{2}
$$

and consequently,

$$
H_{3}=2 a^{2} \sum_{s=1}^{\infty} C_{3} \iint U_{s} d x d y=-2 \pi k \lambda b^{2} a^{2} C_{1}
$$

Summing up, we find the torsional stiffness

$$
H=\pi a^{4}\left\{\left(1-q^{2}\right)^{2}+k p^{4} \lambda^{2}\left(2+\lambda^{2}\right)-2 k p^{2} \lambda\left(2 C_{1}+\lambda\right)+4 q^{2} \Psi_{1}+4 k p^{2} \lambda^{2} \Psi_{2}\right\} / 2 .
$$

The resulting twisting couple is given by

$$
T=\mu \tau H,
$$

where $\mu$ is the modulus of rigidity of the material and $\tau$ is the angle of twist per unit length of the tube.

Stress components. The non-vanishing stress components of the tube are two shear stress components given in terms of the polar coordinates $(r, \phi)$ by the following

$$
z_{r}=\frac{\mu \tau}{a r} \frac{\partial \Psi}{\partial \phi}, \quad z_{\phi}=-\frac{\mu \tau}{a} \frac{\partial \Psi}{\partial r},
$$


while in terms of the polar coordinates $(\rho, \theta)$, they are

$$
z_{\rho}=\frac{\mu \tau}{b \rho} \frac{\partial \Psi}{\partial \theta}, \quad z_{\theta}=-\frac{\mu \tau}{b} \frac{\partial \Psi}{\partial \rho} .
$$

It is now a straightforward matter to find the stress at any point of the cross section. By using appropriate series, the shear stresses on the three boundaries are found as follows:

$$
\begin{aligned}
& {\left[z_{\phi}\right]_{r=1}=\mu \tau a\left(1-2 k \sum_{n=1}^{\infty} n A_{n} \cos n k \phi\right),} \\
& {\left[z_{\theta}\right]_{\rho=\lambda}=\mu \tau a\left(p \lambda+\frac{2}{p \lambda} \sum_{m=1}^{\infty} \frac{m C_{m}}{\lambda^{m}} \cos m \theta\right),} \\
& {\left[z_{\phi}\right]_{r=q}=\mu \tau a\left(q+\frac{2 k}{q} \sum_{n=1}^{\infty} \frac{n B_{n}}{q^{n k}} \cos n k \phi\right) .}
\end{aligned}
$$
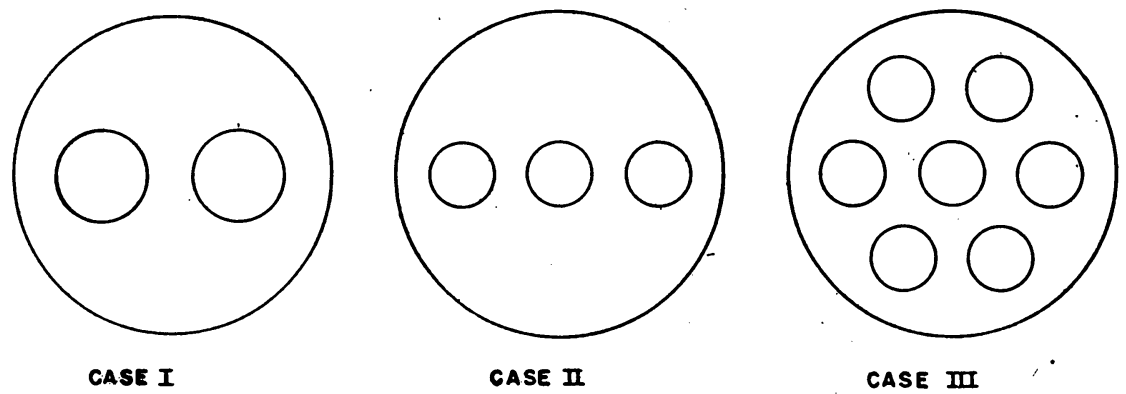

Fig. 3. Illustrative examples.

The maximum shear stress occurs on the boundary across the narrowest sections. Due to the presence of the holes, the greatest shear stress does not necessarily occur on the exterior boundary.

The case without central hole. It is interesting to investigate also the case in which the central hole of the tube does not exist. A solution is obtained for $\psi$ by merely omitting $\psi_{2}$ in Eq. (7), such that

$$
\psi=\psi_{1}+\psi_{3} .
$$

Consequently, the relations obtained from the boundary conditions become

$$
\begin{gathered}
A_{0}=\frac{1}{2}, \quad \Psi_{2}=M_{0}+L_{0}-\frac{1}{2} p^{2}\left(1+\lambda^{2}\right), \\
A_{n}=-P_{n}, \quad \lambda^{-2} C_{1}+M_{1}+L_{1}=p^{2}, \quad \lambda^{-2 m} C_{m}+M_{m}+L_{m}=0,
\end{gathered}
$$

so that the coefficient in (27) is simplified to

$$
{ }^{m} \beta_{s}=-(-1)^{m m} \alpha_{s}+k \sum_{n=1}^{\infty}\left(\begin{array}{c}
n k \\
m
\end{array}\right)\left(\begin{array}{c}
n k-1 \\
s-1
\end{array}\right) p^{2 n k} .
$$


Numerical examples. The foregoing method of solution will be illustrated by working out the following three examples:

\begin{tabular}{|c|c|c|c|c|c|}
\hline Case & $k$ & $p$ & $q$ & $\lambda$ & No. of Holes \\
\hline I & 2 & $\frac{3}{7}$ & 0 & $\frac{2}{3}$ & 2 \\
\hline II & 2 & $\frac{3}{5}$ & $\frac{1}{5}$ & $\frac{1}{3}$ & 3 \\
\hline III & 6 & $\frac{3}{5}$ & $\frac{1}{5}$ & $\frac{1}{3}$ & 7 \\
\hline
\end{tabular}

The arrangements are shown in Fig. 3. In each case the holes have equal radii and the nearest distance between any two adjacent boundaries is equal to the radius of one hole. These cases probably represent the most crucial ones ever met in practice. Tables of the coefficients ${ }^{m} \beta_{s}$ are first prepared, with which the parametric coefficients $C_{m}$ are found by the method of successive approximations; the results being shown in Appendix B and in the accompanying Table I. The shear stresses along the boundaries are then computed and shown in Table II. Fig. 4 shows the results diagrammatically.

TABLE I. The coefficients $C_{m}$

\begin{tabular}{c|r|r|r}
\hline \hline$m$ & \multicolumn{1}{|c|}{ Case I } & \multicolumn{1}{c}{ Case II } & \multicolumn{1}{c}{ Case III } \\
\hline 1 & $7.8560 \times 10^{-2}$ & $4.2392 \times 10^{-2}$ & $3.0593 \times 10^{-2}$ \\
2 & $3.1549 \times 10^{-3}$ & $3.1240 \times 10^{-4}$ & $5.8718 \times 10^{-4}$ \\
3 & $-3.0714 \times 10^{-4}$ & $1.9115 \times 10^{-5}$ & $4.9273 \times 10^{-5}$ \\
4 & $9.6791 \times 10^{-5}$ & $3.2509 \times 10^{-7}$ & $-7.4587 \times 10^{-6}$ \\
5 & $-1.7036 \times 10^{-5}$ & $1.8686 \times 10^{-7}$ & $4.9622 \times 10^{-7}$ \\
6 & $3.9811 \times 10^{-6}$ & $-1.2587 \times 10^{-8}$ & $5.9963 \times 10^{-8}$ \\
7 & $-8.0836 \times 10^{-7}$ & $2.5340 \times 10^{-9}$ & $-1.0635 \times 10^{-8}$ \\
8 & $1.7422 \times 10^{-7}$ & $-2.7810 \times 10^{-10}$ & $4.7674 \times 10^{-10}$ \\
\hline
\end{tabular}

TABLE II. Shear stress/ $\mu \tau a$ along boundaries.

\begin{tabular}{|c|c|c|c|c|c|c|c|c|}
\hline \multirow{2}{*}{$\begin{array}{c}k \phi \\
\text { or } \\
\theta\end{array}$} & \multicolumn{2}{|c|}{ Case I } & \multicolumn{3}{|c|}{ Case II } & \multicolumn{3}{|c|}{ Case III } \\
\hline & $\begin{array}{c}\text { Exterior } \\
\text { boundary }\end{array}$ & $\begin{array}{c}\text { Eccentric } \\
\text { holes }\end{array}$ & $\begin{array}{r}\text { Exterior } \\
\text { boundary }\end{array}$ & $\begin{array}{c}\text { Eccentric } \\
\text { holes }\end{array}$ & $\begin{array}{c}\text { Central } \\
\text { hole }\end{array}$ & $\begin{array}{c}\text { Exterior } \\
\text { boundary }\end{array}$ & $\begin{array}{c}\text { Eccentric. } \\
\text { holes }\end{array}$ & $\begin{array}{c}\text { Central } \\
\text { hole }\end{array}$ \\
\hline 0 & 1.185 & 1.194 & 1.308 & 1.547 & 0.459 & 1.127 & 1.247 & 0.214 \\
\hline $30^{\circ}$ & 1.125 & 1.049 & 1.124 & 1.327 & 0.402 & 1.105 & 1,053 & 0.212 \\
\hline $60^{\circ}$ & 1.022 & 0.660 & 0.967 & 0.793 & 0.277 & 1.050 & 0.583 & 0.207 \\
\hline $90^{\circ}$ & 0.956 & 0.198 & 0.930 & 0.145 & 0.160 & 0.988 & 0.068 & 0.200 \\
\hline $120^{\circ}$ & 0.928 & -0.199 & 0.928 & -0.451 & 0.081 & 0.938 & -0.259 & 0.193 \\
\hline $150^{\circ}$ & 0.918 & -0.396 & 0.931 & -0.871 & 0.039 & 0.907 & -0.529 & 0.188 \\
\hline $180^{\circ}$ & 0.916 & -0.392 & 0.932 & -1.033 & 0.026 & 0.897 & -0.678 & 0.186 \\
\hline
\end{tabular}

It appears that in all the cases the greatest shear stress occurs at those points on the boundaries of the holes which are nearest to the exterior boundary. The magnitudes are equal to $1.194,1.547$ and 1.247 respectively except for a factor $\mu \tau a$. The points of greatest shear stress will, however, shift to the exterior boundary if the eccentric holes are sufficiently remote from the exterior boundary. It is seen that in each case there exist two null points or points of zero stress on the boundary of each hole at an angle $\theta$ numerically between $90^{\circ}$ and $120^{\circ}$ or, to be more precise, at $105^{\circ}$, 


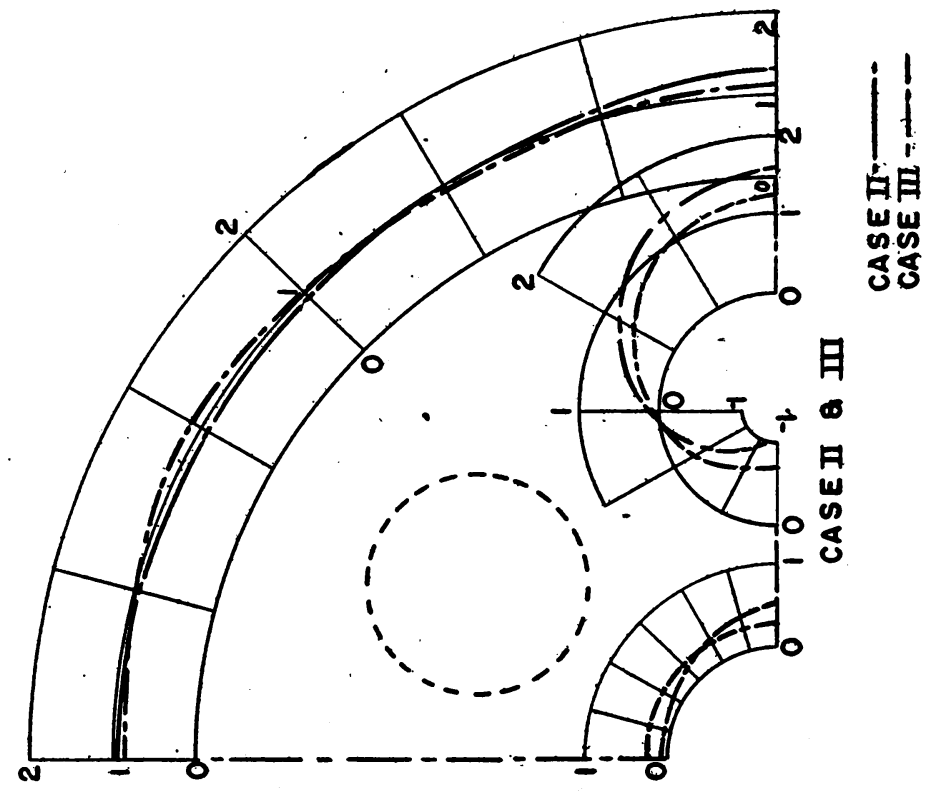

递

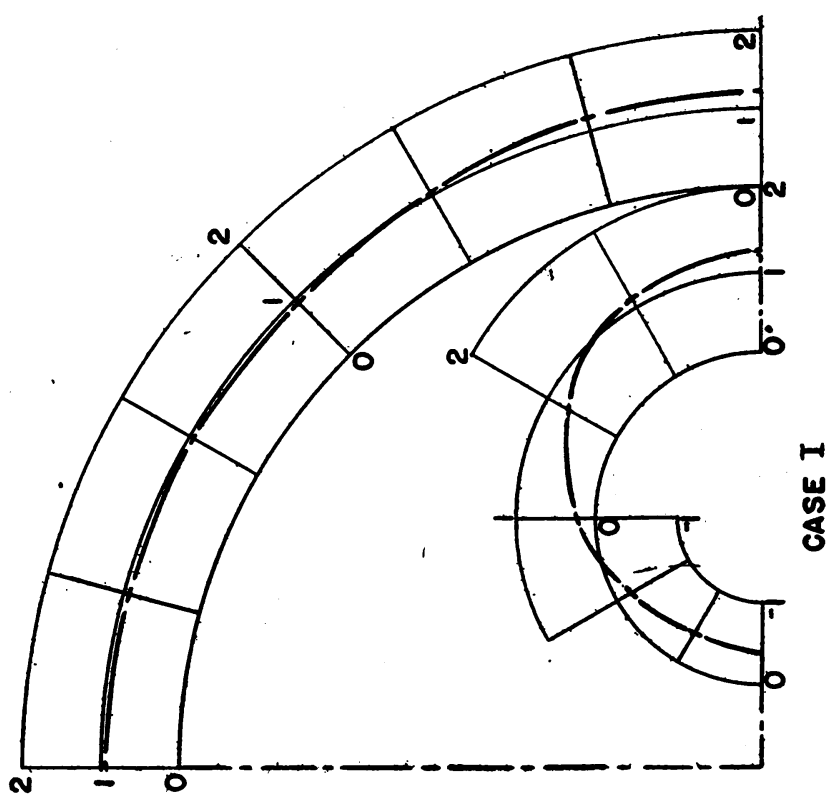


$96^{\circ}$ and $95^{\circ}$ respectively. It is also seen that due to the presence of holes the maximum shear stress on the exterior boundary is increased by $18.5 \%, 30.8 \%$ and $12.7 \%$ respectively. From the stress distribution on the boundaries it appears that among the three cases the case II is the most unfavorable arrangement in which, it is recalled, the centers of the holes are situated collinearly on the same diameter of the section. The stress distribution on the boundary of the central hole is less important since the magnitude is smaller.

Table III shows the cross-sectional area $A$ and the torsional stiffness $H$ of the tube, in which the last two columns indicate their percentage reductions due to the presence of holes respectively. The relative reductions in torsion stiffness due to $1 \%$ reduction in area are equal to $0.729 \%, 1.589 \%$ and $0.963 \%$ respectively. Again, it appears that the case II is the most unfavorable arrangement among the three where the effectiveness of torsion modulus is concerned.

TABLE III. Cross-sectional area $A$ and torsional stiffness $H$.

\begin{tabular}{r|c|c|c|c}
\hline \hline Case & $A / \pi a^{2}$ & $H / \frac{1}{2} \pi a^{4}$ & \% reduction of $A$ & \% reduction of $H$ \\
\cline { 2 - 4 } I & 0.8367 & 0.8809 & 16.33 & 11.91 \\
II & 0.8800 & 0.8093 & 12.00 & 19.07 \\
III & 0.7200 & 0.7305 & 28.00 & 26.95 \\
\hline
\end{tabular}

The author wishes to express his deep appreciation to Dr. H. S. Tsien of the Guggenheim Aeronautical Laboratory, California Institute of Technology, for helpful criticism and suggestions.

\section{APPENDIX A}

Class of functions invariant with respect to rotation. Let there be on the circle $|z|=b$ in the Argand plane a series of $k$ points

$$
z=b e^{2 m \pi i / k}
$$

where $m=0,1,2, \cdots,(k-1)$.

A function with a logarithmic singularity at each such point is defined by

$$
W_{0}=-\log \left(z^{k}-b^{k}\right)=-\log (z-b)-\sum_{m=1}^{k-1} \log \left(z-b e^{2 m \pi i / k}\right) .
$$

Writing $z-b=b \zeta$, we have except for a constant

$$
W_{0}=-\log \zeta-\sum_{m=1}^{k-1} \log \left(1-u_{m} \zeta\right),
$$

where

$$
1 / u_{m}=1-e^{2 m \pi i / k}
$$

such that $u_{m}$ is a root of the equation

$$
(u-1)^{k}-u^{k}=0 .
$$

Expansion yields

$$
W_{0}=-\log \zeta+\sum_{n=1}^{\infty}(-1)^{n}{ }^{n} \alpha_{0} \zeta^{n},
$$


where

$$
{ }^{n} \alpha_{0}=\frac{1}{n} \sum_{m=1}^{k-1} u_{m}^{n}=\sigma_{n} / n,
$$

$\sigma_{n}$ denoting the sum of the $n$th power of the roots of Eq. (A.4), which may be calculated for any given values of $k$ and $n$.

It is readily shown that functions derived from Eq. (A.2) by differentiation with respect to $b$ are invariant with respect to rotation about the origin through angles $2 m \pi / k$. If we define

$$
W_{s}=\frac{b^{s}}{(s-1) !} \frac{d^{s} W_{0}}{d b^{s}},
$$

we find

$$
W_{s}=\frac{1}{\zeta^{s}}+\sum_{m=1}^{k-1} \frac{\left(1-u_{m}^{-1}\right)^{s}}{\left(u_{m}^{1-}+\zeta\right)^{s}}=\frac{1}{\zeta^{s}}+\sum_{m=1}^{k-1}\left(u_{m}-1\right)^{s} \sum_{n=0}^{\infty}(-1)^{n}\left(\begin{array}{c}
n+s-1 \\
n
\end{array}\right)\left(u_{m} \zeta\right)^{n},
$$

or

$$
W_{s}=\frac{1}{\zeta^{s}}+\sum_{n=0}^{\infty}(-1)^{n}{ }^{n} \alpha_{s} \zeta^{n}
$$

where

$$
\begin{aligned}
{ }^{n} \alpha_{s} & =\left(\begin{array}{c}
n+s-1 \\
n
\end{array}\right) \sum_{m=1}^{k-1}\left(u_{m}-1\right)^{s} u_{m}^{n}=\left(\begin{array}{c}
n+s-1 \\
n
\end{array}\right) \sum_{t=0}^{s}(-1)^{t}\left(\begin{array}{l}
s \\
t
\end{array}\right) \sigma_{n+s-t} \\
& =\left(\begin{array}{c}
n+s-1 \\
n
\end{array}\right) \Delta^{s} \sigma_{n},
\end{aligned}
$$

$\Delta^{*} \sigma_{n}$ being the $s$ th finite difference of the series $\sigma_{n}, \sigma_{n+1}, \sigma_{n+2}, \ldots$ From Eq. (A.4) we obtain

$$
u^{n+m k}=\sum_{t=0}^{m k}(-1)^{t}\left(\begin{array}{c}
m k \\
t
\end{array}\right) u^{n+m k-t},
$$

where $m$ is an integer. Summing over the roots,

$$
\sigma_{n+m k}=\sum_{t=0}^{m k}(-1)^{\imath}\left(\begin{array}{c}
m k \\
t
\end{array}\right) \sigma_{n+m k-t}=\Delta^{m k} \sigma_{n} .
$$

Hence

$$
\Delta^{m k+s} \sigma_{n}=\Delta^{s} \Delta^{m k} \sigma_{n}=\Delta^{s} \sigma_{n+m k},
$$

so that all the coefficients can be found from the values of $\sigma_{n}$ and its first $(k-1)$ th finite differences.

Now, if we define

$$
W_{0}=U_{0}-i V_{0}, \quad W_{8}=U_{8}-i V_{8}
$$

and $\zeta=\rho e^{i \theta}$, we find by equating the real and imaginary parts 


$$
\begin{aligned}
& U_{0}=-\log \rho+\sum_{n=1}^{\infty}(-1)^{n}{ }^{n} \alpha_{0} \rho^{n} \cos n \theta \\
& V_{0}=\theta-\sum_{n=1}^{\infty}(-1)^{n}{ }^{n} \alpha_{0} \rho^{n} \sin n \theta \\
& U_{s}=\frac{\cos s \theta}{\rho^{8}}+\sum_{n=0}^{\infty}(-1)^{n}{ }^{n} \alpha_{s} \rho^{n} \cos n \theta \\
& V_{s}=\frac{\sin s \theta}{\rho^{8}}=\sum_{n=1}^{\infty}(-1)^{n}{ }^{n} \alpha_{s} \rho^{n} \sin n \theta
\end{aligned}
$$

\begin{tabular}{|c|c|c|c|c|}
\hline$s$ & $m=1$ & $m=2$ & $m=3$ & $m=4$ \\
\hline 1 & $-1.0547 \times 10^{-1}$ & $2.0736 \times 10^{-1}$ & $-5.1703 \times 10^{-2}$ & $3.4879 \times 10^{-2}$ \\
\hline 2 & $4.1472 \times 10^{-1}$ & $-7.2751 \times 10^{-2}$ & $1.6111 \times 10^{-1}$ & $-6.4085 \times 10^{-2}$ \\
\hline 3 & $-1.5511 \times 10^{-1}$ & $2.4167 \times 10^{-1}$ & $-1.1142 \times 10^{-1}$ & $1.4009 \times 10^{-1}$ \\
\hline 4 & $1.3952 \times 10^{-1}$ & $-1.2817 \times 10^{-1}$ & $1.8679 \times 10^{-1}$ & $-1.1476 \times 10^{-1}$ \\
\hline 5 & $-7.4973 \times 10^{-2}$ & $1.2605 \times 10^{-1}$ & $-1.2240 \times 10^{-1}$ & $1.5170 \times 10^{-1}$ \\
\hline 6 & $4.7899 \times 10^{-2}$ & $-7.8758 \times 10^{-2}$ & $1.1563 \times 10^{-1}$ & $-1.1495 \times 10^{-1}$ \\
\hline 7 & $-2.7106 \times 10^{-2}$ & $5.5633 \times 10^{-2}$ & $-7.7962 \times 10^{-2}$ & $1.0623 \times 10^{-1}$ \\
\hline 8 & $1.5692 \times 10^{-2}$ & $-3.4860 \times 10^{-2}$ & $5.9407 \times 10^{-2}$ & $-7.9025 \times 10^{-2}$ \\
\hline$s$ & $m=5$ & $m=6$ & $m=7$ & $m=8$ \\
\hline 1 & $-1.4995 \times 10^{-2}$ & $7.9832 \times 10^{-3}$ & $-3.8723 \times 10^{-3}$ & $1.9615 \times 10^{-3}$ \\
\hline 2 & $5.0421 \times 10^{-2}$ & $-2.6253 \times 10^{-2}$ & $1.5891 \times 10^{-2}$ & $-8.7149 \times 10^{-8}$ \\
\hline 3 & $-7.3439 \times 10^{-2}$ & $5.7816 \times 10^{-2}$ & $-3.4184 \times 10^{-2}$ & $2.2278 \times 10^{-2}$ \\
\hline 4 & $1.1797 \times 10^{-1}$ & $-7.8946 \times 10^{-2}$ & $5.5966 \times 10^{-2}$ & $-3.9978 \times 10^{-2}$ \\
\hline 5 & $-1.1196 \times 10^{-1}$ & $1.0893 \times 10^{-1}$ & $-7.7443 \times 10^{-2}$ & $6.1346 \times 10^{-2}$ \\
\hline 6 & $1.3072 \times 10^{-1}$ & $-1.0718 \times 10^{-1}$ & $1.0010 \times 10^{-1}$ & $-7.7385 \times 10^{-2}$ \\
\hline 7 & $-1.0842 \times 10^{-1}$ & $1.1678 \times 10^{-1}$ & $-1.0180 \times 10^{-1}$ & $9.3473 \times 10^{-2}$ \\
\hline 8 & $9.8847 \times 10^{-2}$ & $-1.0237 \times 10^{-1}$ & $1.0703 \times 10^{-1}$ & $-9.6648 \times 10^{-2}$. \\
\hline
\end{tabular}

APPENDIX B. TABLES OF COEFFICIENTS ${ }^{m} \beta_{s}$

CASE I. $k=2, p=3 / 7, q=0$

CASE II. $k=2, p=3 / 5, q=1 / 5$

\begin{tabular}{l|l|l|l|l}
\hline \hline$s$ & $m=1$ & $m=2$ & $m=3$ & \multicolumn{1}{|c}{$m=4$} \\
\cline { 2 - 4 } 1 & $4.9867 \times 10^{-1}$ & $5.8617 \times 10^{-1}$ & $3.2014 \times 10^{-1}$ & $4.0272 \times 10^{-2}$ \\
2 & 1.1751 & 1.3668 & $9.7830 \times 10^{-1}$ & 1.2483 \\
3 & $9.6041 \times 10^{-1}$ & 1.4633 & 2.9506 & 1.7326 \\
4 & $1.6086 \times 10^{-1}$ & 2.4953 & 2.3101 & 6.1916 \\
5 & 1.2869 & $4.4025 \times 10^{-1}$ & 5.6549 & 4.1153 \\
6 & $-9.9512 \times 10^{-1}$ & 3.1571 & $9.7328 \times 10^{-1}$ & $1.2122 \times 10$ \\
7 & 2.0139 & -1.6578 & 7.6527 & 2.5465 \\
8 & -2.3208 & 4.4190 & -2.9699 & $1.7278 \times 10$ \\
\hline
\end{tabular}




\begin{tabular}{c|l|l|l|l}
\hline$s$ & $m=5$ & \multicolumn{1}{|c|}{$m=6$} & \multicolumn{1}{c}{$m=7$} & $m=8$ \\
\cline { 2 - 4 } 1 & $2.5737 \times 10^{-1}$ & $-1.6585 \times 10^{-1}$ & $2.8770 \times 10^{-1}$ & $-2.9011 \times 10^{-1}$ \\
2 & $1.7611 \times 10^{-1}$ & 1.0524 & $-4.6363 \times 10^{-1}$ & 1.1047 \\
3 & 3.3929 & $4.8664 \times 10^{-1}$ & 3.3914 & -1.1137 \\
4 & 3.2923 & 8.0811 & 1.4551 & 8.6392 \\
5 & $1.2917 \times 10$ & 6.6271 & $1.8327 \times 10$ & 4.0214 \\
6 & 7.9600 & $2.6963 \times 10$ & 9.7852 & $4.0734 \times 10$ \\
7 & $2.5212 \times 10$ & $1.6097 \times 10$ & $5.6595 \times 10$ & $2.9178 \times 10$ \\
8 & 7.3042 & $7.4312 \times 10$ & $2.6870 \times 10$ & $1.1962 \times 10^{2}$ \\
\hline
\end{tabular}

CASE III. $k=6, p=3 / 5, q=1 / 5$

\begin{tabular}{c|c|c|c|c}
\hline \hline$s$ & $m=1$ & $m=2$ & $m=3$ & $m=4$ \\
\cline { 2 - 4 } 1 & -2.8379 & 1.6559 & 1.0945 & -1.7600 \\
2 & 3.3118 & -5.8522 & 5.9408 & 5.8842 \\
3 & 3.2836 & 8.9112 & $-1.7916 \times 10$ & $1.8392 \times 10$ \\
4 & -7.0400 & $1.1769 \times 10$ & $2.4523 \times 10$ & $-6.6469 \times 10$ \\
5 & 5.6284 & $-2.8311 \times 10$ & $3.8057 \times 10$ & $7.6789 \times 10$ \\
6 & 6.0363 & $2.2409 \times 10$ & $-1.0871 \times 10^{2}$ & $1.3393 \times 10^{2}$ \\
7 & $-1.3620 \times 10$ & $2.8477 \times 10$ & $8.8391 \times 10$ & $-4.1222 \times 10^{9}$ \\
8 & 7.9354 & $-7.0520 \times 10$ & $1.2148 \times 10^{2}$ & $3.4151 \times 10^{2}$ \\
& & & & \\
$s$ & $m=5$ & $m=6$ & $m=7$ & $m=8$ \\
\hline 1 & 1.1569 & 1.0060 & -1.9457 & $9.9193 \times 10^{-1}$ \\
2 & $-1.1324 \times 10$ & 7.4698 & 8.1366 & $-1.7630 \times 10$ \\
3 & $2.2834 \times 10$ & $-5.4335 \times 10$ & $3.7881 \times 10$ & $4.5598 \times 10$ \\
4 & $6.1432 \times 10$ & $8.9290 \times 10$ & $-2.3555 \times 10^{2}$ & $1.7075 \times 10^{2}$ \\
5 & $-2.4343 \times 10^{2}$ & $2.2150 \times 10^{2}$ & $3.4389 \times 10^{2}$ & $-9.8099 \times 10^{2}$ \\
6 & $2.6581 \times 10^{2}$ & $-9.0308 \times 10^{2}$ & $8.1328 \times 10^{2}$ & $1.3199 \times 10^{3}$ \\
7 & $4.8145 \times 10^{2}$ & $9.4883 \times 10^{2}$ & $-3.3856 \times 10^{3}$ & $3.0419 \times 10^{3}$ \\
8 & $-1.5696 \times 10^{3}$ & $1.7598 \times 10^{3}$ & $3.4764 \times 10^{3}$ & $-1.2761 \times 10^{4}$ \\
\hline
\end{tabular}

